\title{
Transient cortical blindness after heart surgery in a child patient -A case report-
}

\author{
Young Duck Shin ${ }^{1}$, Seung Woon Lim ${ }^{1}$, Jin Ho Bae ${ }^{1}$, Dong Hun Lee ${ }^{1}$, Dhong Hion Baek ${ }^{1}$, and Jang Soo Hong ${ }^{2}$ \\ Departments of ${ }^{1}$ Anesthesiology and Pain Medicine, ${ }^{2}$ Thoracic and Cardiovascular Surgery, College of Medicine, Chungbuk National \\ University, Cheongju, Korea
}

Visual loss occurring after pediatric cardiac surgery employing cardiopulmonary bypass (CPB) is relatively rare but the risk is substantial. Compromised cerebral perfusion due to a CPB related micro-embolization and inflammatory vascular changes as well as reduced oxygen carrying capacity in hemodilution and hypothermia during CPB might be major contributing factors to the development of postoperative visual loss after cardiac surgery with CPB. A case of immediate but transient postoperative visual loss was encountered in a 21-month-old male who underwent tricuspid valve surgery. Despite routine intraoperative measures to maintain an adequate perfusion pressure throughout the procedure, postoperative computed tomography revealed a subacute infarct in his occipital lobe. Recovery began on postoperative day 28, and the patient's vision was restored by 31 days. (Korean J Anesthesiol 2010; 59: 61-64)

Key Words: Blindness, Cardiac surgical procedures, Complications.

Visual loss after general anesthesia is a rare complication caused by a range of factors [1-4]. Although no improper positioning placed pressure on the eye-area [4], damage to the occipital cortex after a long surgery or extracorporeal circulation in cardiac surgery was reported to occasionally cause visual loss $[2,5]$. However, the diagnosis can be delayed when it does occur in pediatric patients because they usually are unable to express themselves clearly. We report a pediatric patient who had undergone cardiopulmonary bypass (CPB) surgery and experienced 4 weeks of cortical blindness before natural healing occurred.

\section{Case Report}

A 21-month-old male patient, $75 \mathrm{~cm}$ in height and $10 \mathrm{~kg}$ in weight, experienced tricuspid valve regurgitation (TVR) and was scheduled to undergo tricuspid valve plasty. The TVR was discovered in a follow-up test 1 year after he had undergone ventricular septal defect repair surgery. Before tricuspid valve plasty, a TEE was performed, which showed a level 3-4 TVR and severe right ventricular hypertrophy. There were no other abnormal findings.

The patient was given no preoperative treatment before he

Received: August 27, 2009. Revised: 1st, September 18, 2009; 2nd, October 20, 2009. Accepted: November 24, 2009.

Corresponding author: Seung Woon Lim, M.D., Department of Anesthesiology and Pain Medicine, College of Medicine, Chungbuk National University, 62, Gaesin-dong, Heungdeok-gu, Cheongju 361-711, Korea. Tel: 82-43-269-6237, Fax: 82-43-272-0264, E-mail: swlim@chungbuk.ac.kr This work was supported by Chungbuk National University Grant in 2009.

(c) This is an open-access article distributed under the terms of the Creative Commons Attribution Non-Commercial License (http:// creativecommons.org/licenses/by-nc/3.0/), which permits unrestricted non-commercial use, distribution, and reproduction in any medium, provided the original work is properly cited. 
Table 1. Perioperative Arterial Blood Gas Analysis and Hematocrit

\begin{tabular}{|c|c|c|c|c|c|}
\hline & During bypass & Weaning period & After CPB & $1 \mathrm{~h}$ after ICU arrival & $24 \mathrm{~h}$ after ICU arrival \\
\hline $\mathrm{FiO}_{2}$ & 1.0 & 1.0 & 0.8 & 0.4 & 0.4 \\
\hline $\mathrm{pH}$ & 7.406 & 7.369 & 7.318 & 7.435 & 7.428 \\
\hline $\mathrm{PaCO}_{2}(\mathrm{mmHg})$ & 39.2 & 30.2 & 44.5 & 33.0 & 32.6 \\
\hline $\mathrm{PaO}_{2}(\mathrm{mmHg})$ & 419 & 323 & 236 & 152 & 168 \\
\hline $\mathrm{BE}$ & 0.0 & -7.2 & -3.0 & -1.8 & -2.5 \\
\hline $\mathrm{HCO}_{3}^{-}$ & 24.1 & 17.0 & 22.2 & 21.8 & 21.2 \\
\hline $\mathrm{SaO}_{2}(\%)$ & 99.7 & 99.5 & 99.2 & 99.2 & 99.2 \\
\hline Hct $(\%)$ & 27.5 & 21.3 & 24.7 & 27.6 & 30.7 \\
\hline
\end{tabular}

CPB: cardiopulmonary bypass, ICU: intensive care unit, $\mathrm{FiO}_{2}$ : inspired oxygen fraction, Hct: hematocrit.

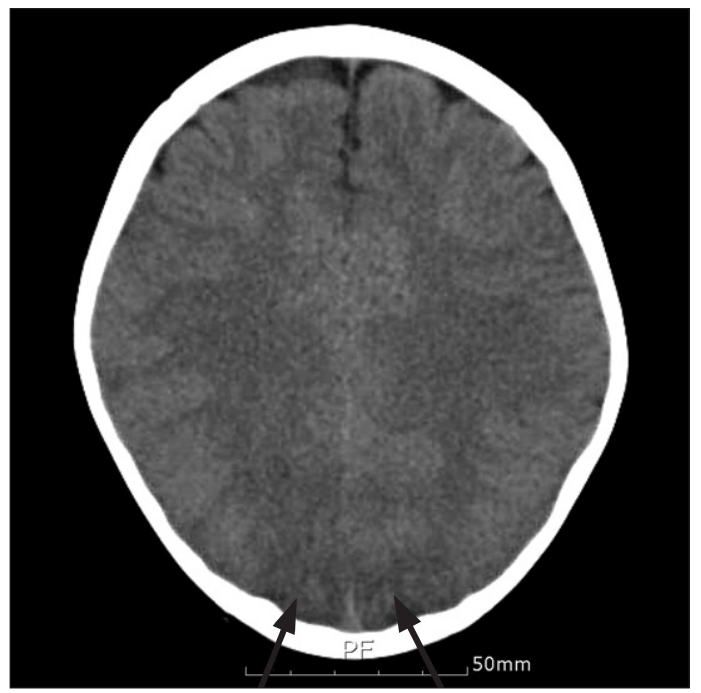

Fig. 1. Brain computed tomography shows an infarction in the occipital lobe (arrows).

was transferred to the OR. The ECG machine, NIBP and pulse oximeter $\mathrm{SpO}_{2}$ were attached. Atropine, thiopental sodium, and vecuronium were administered to induce anesthesia and muscle relaxation. Regulated assisted respiration was secured with sevoflurane 8 vol\%. Endotracheal intubation was performed using a $4.5 \mathrm{~mm}$ internal diameter tube. The anesthesia was sustained with $50 \% \mathrm{O}_{2}$ and $2-3$ vol\% sevoflurane. A catheter was placed at the left radial artery to measure the constant blood pressure and analyze the ABGA (Table 1). A central vein catheter was intubated in the right subclavian vein. A membrane oxygenator and roller pump were used in the cardiopulmonary bypass machine. A 12 Fr cannula was placed in the aorta for the cardiopulmonary bypass. There were complications due to the adhesion of the SVC and IVC near the surgical site. Therefore, a $20 \mathrm{Fr}$ and $22 \mathrm{Fr}$ cannula was placed on them, respectively. $2 \mathrm{U}$ of packed red blood cells (PRC), albumin, and mannitol was used as the priming solution. Immediately after activating the cardiopulmonary bypass, the measured hematocrit level was $27.5 \%$. The acid-base balance management mid-surgery was performed with an $\alpha$-stat. During extracorporeal circulation, the body temperature was aimed at a moderately low degree of $25^{\circ} \mathrm{C}$. The perfusion rate was kept at $0.5-1.0 \mathrm{~L} / \mathrm{min}$. The blood pressure was $45-60$ mmHg. After tricuspid valve surgery, the patient was weaned from the cardiopulmonary bypass by reducing the perfusion rate of the machine and starting mechanical ventilation. Whilst reducing the perfusion rate, dopamine and dobutamine was administered continuously. The total anesthesia, surgery and extracorporeal circulation time was 510 minutes, 420 minutes and 180 minutes, respectively. The amount of blood loss was estimated to be $800 \mathrm{ml}$. During surgery, $600 \mathrm{ml}$ of a crystalloid solution, 3 U PRC and 2 U platelets were administered. The urine output was $200 \mathrm{ml}$.

Two days after the surgery, the endotracheal tube was extubated. However the patient's eyes could not focus, and his upper and lower limbs experienced intermittent convulsions. Brain computed tomography revealed findings indicative of a cerebral infarction in the occipital lobe (Fig. 1). Visual loss was suspected due to the convulsions and cortical damage. EEG was performed, which showed no abnormal findings of alpha rhythm loss, which is customary in cortical blindness. The general convulsions were no longer observed. Phenobarbital was administered until 19 days after surgery under the suspicion of a connection between the ocular deviation and the convulsions. The convulsions did not return, but the reaction to light or objects in front of the eyes did not trigger an avoidance response from the patient. There were no hopeful clinical signs of a visual recovery but fundoscopy showed normal findings. On the postoperative day 28, when the flash visual evoked potential (FVEP) and MRI had been planned, the patient's eyes refocused. He was able to recognize the objects in front of his eyes, could walk and avoid things in his path, and was able to recognize his guardians. On postoperative day 31 , his eyesight had returned to normal. We wanted to run objective back-up tests but the guardians refused. The patient was discharged on postoperative day 42 . 


\section{Discussion}

Post surgery visual loss or reduction can be caused by a variety of factors, and occurs $0.1 \%$ to $1 \%$ of cases [6]. Visual loss is caused by damage to any connecting striate area between the cornea and occipital cortex. Direct pressure on the eyeball, indirect increase in ocular tension due to improper positioning and blood vessel damage can cause central retinal artery occlusion or ischemic optic nerve damage, which can lead to visual loss $[1,7]$. In addition, a severe decrease in blood pressure, emboli, and thrombus mid- or post-surgery can cause an infarction in the occipital cortex, which can lead to cortical blindness $[8,9]$. Post-surgery visual loss can be caused by a range of factors. One of the causes of cortical blindness is extracorporeal circulation during cardiac surgery [2]. Visual loss after a CPB might be caused primarily due to emboli, severe low blood pressure, acute anemia, hypoxia or a combination of these factors $[3,7]$. In the present case, emboli appeared to be the most likely cause. William et al. [7] examined 92 patients who experienced visual loss after undergoing cardiac and noncardiac surgery. Twenty seven cases were cortical blindness, of which 22 cases occurred after cardiac surgery (81\%). Aldrich et al. [3] reported a retrospective study of 25 patients, where the causes of cortical blindness were natural ischemic stroke in 8 patients (32\%), cardiac surgery in 5 patients $(20 \%)$, cerebral angiography in 3 patients (12\%), non-surgery in 4 patients (16\%), seizure in 2 patients ( $8 \%$ ), and other factors, such as damage to the head and peritoneal dialysis, in 3 patients (12\%). There were also cases of temporary cortical blindness reported after postcardiac surgery angiography [8].

In the case of children, the early indicators of brain damage after surgery are age, the complexity of surgery, metabolic acidosis, increase in lactate, and artery acidosis [9]. Diagnosing cortical blindness is simple when the patient makes a complaint but as in our case, a pediatric patient has limited ability to communicate. Therefore, conducting an EEG, FVEP, angiography, and funduscopy can assist in making a diagnosis [3]. In this case, the EEG did not show the abnormal finding of a loss in alpha rhythm. However, computed laminography revealed an infarction in the occipital cortex. The patient was uncooperative with the funduscopy and there was ocular deviation. Hence, immediate assessments could not be made. Three days after surgery, a funduscopy was performed and showed normal findings. We checked for visual loss with light and objects to test the patient's avoidance response but he did not respond properly. The patient was unable to avoid objects when walking.

The improvement in surgical methods has led to fewer cases of large emboli. However, microemboli caused by extracorporeal circulation can obstruct the blood flow in the cerebral vessels, particularly in the watershed area [10]. Braekken et al. [11] reported that cardiac surgery using extracorporeal circulation causes microemboli in most cases. In addition, in patients with neural damage, there are more cases of transcranial Doppler detection of microembolic signals in the right middle cerebral artery than in patients without. Microemboli midsurgery occurs most often when blocking the aorta (18\%) and unblocking the aorta (13\%). Rodriguez and Belway [12] stated that in pediatric cardiac surgery, the likelihood of microemboli can increase with increasing time of extracorporeal circulation depending on the oxygenator, circuit, adnexa parts, and components. The surgery in this case was not complicated, but may have increased the likelihood of microembolization due to the long extracorporeal circulation time.

Another cause is an impairment in brain function due to the cardiopulmonary bypass. $\alpha$-stat was used in the acidbase balance management of the presented case. However, we should have been more careful in the selecting the $\alpha$-stat and pH-stat method. Deep hypothermic circulatory arrest was not performed. Nevertheless, the strength of the $\mathrm{pH}$-stat should have been considered regarding neural sequelae. The СРВ priming solution in pediatric patients, unlike adults, can cause blood thinning, which can lead to possible ischemic organ failure.

Aldrich et al. [3] reported that conditions, such as the age below 40 , no diabetes or high blood pressure, and no impairment in cognitive skills, language skills or memory, indicate a good prognosis for visual recovery. In addition, EEG tests can assist in a diagnosis but are not helpful in accurately predicting the prognosis. In this study, the patient was young and the EEG tests were normal. In addition, he did not have diabetes or any other systemic diseases. Two weeks after the surgery, he recovered his speech and cognitive skills, so we did expected a good prognosis. The patient's vision recovered rapidly from postoperative day 28 and he could be discharged. Other tests such as a FVEP were not performed, so the precise extent of the recovery rate is unknown. A follow-up observation will be needed to obtain this information.

Considerable effort is required to reduce neural sequelae after pediatric cardiac surgery, such as visual loss. Active acid-base balance management is needed. The CPB machine's circuit components and type choice should be made on what can best prevent the likelihood of emboli. A pediatric transesophageal scan and cerebral oxymeter can help in the early identification of neural side-effects. A transesophageal scan plays an important role in eliminating emboli, so it should be prepared for all suitable pediatric patients. The pathological difference between an adult and pediatric cardiopulmonary bypass should be recognized, and proper measures should be followed. Postsurgery cerebrovascular damage should be managed carefully. 
Seizures can interfere with oxygen delivery, so rapid, active treatment is needed. When cortical blindness is suspected, the ophthalmologist and neurologist should perform a combined examination, being cautious of permanent sequelae.

In conclusion, with the reported visual loss in our pediatric patient, surgeons should consider the possibility of visual loss and cerebrovascular injury after a cardiac surgery using cardiopulmonary bypass. Considerable effort should be made to prevent emboli and thrombus formation during surgery. Post-surgery seizures and low blood pressure should be corrected immediately. In addition, any cerebrovascular disorders and symptoms should be observed carefully.

\section{References}

1. Warner ME, Warner MA, Garrity JA, MacKenzie RA, Warner DO. The frequency of perioperative vision loss. Anesth Analg 2001; 93: 1417-21.

2. Bagheri J, Mandegar MH, Sarzaeem MR, Chitsaz S. Transient bilateral cortical visual loss after coronary artery bypass grafting in a normotensive risk-free patient. Heart Surg Forum 2008; 11: E24851.

3. Aldrich MS, Alessi AG, Beck RW, Gilman S. Cortical blindness: etiology, diagnosis, and prognosis. Ann Neurol 1987; 21: 149-58.

4. Yang EW, Lee DH, Park DP. Visual loss after cervical spine surgery in the prone position. Korean J Anesthesiol 2003; 45: 419-21.

5. Ahn KR, Choi JK, Kim CS, Kang KS, Kim JE, Yoo SH, et al. Bilateral cortical blindness due to parieto-occipital infarction after general anesthesia. Korean J Anesthesiol 2004; 47: 122-5.

6. Shahian DM, Speert PK. Symptomatic visual deficits after open heart operations. Ann Thorac Surg 1989; 48: 275-9.

7. Williams EL, Hart WM Jr, Tempelhoff R. Postoperative ischemic optic neuropathy. Anesth Analg 1995; 80: 1018-29.

8. Kamata J, Fukami K, Yoshida H, Mizunuma Y, Moriai N, Takino T, et al. Transient cortical blindness following bypass graft angiography. A case report. Angiology 1995; 46: 937-46.

9. Trittenwein G, Nardi A, Pansi H, Golej J, Burda G, Hermon M, et al. Early postoperative prediction of cerebral damage after pediatric cardiac surgery. Ann Thorac Surg 2003; 76: 576-80.

10. Braekken SK, Reinvang I, Russell D, Brucher R, Svennevig JL. Association between intraoperative cerebral microembolic signals and postoperative neuropsychological deficit: comparison between patients with cardiac valve replacement and patients with coronary artery bypass grafting. J Neurol Neurosurg Psychiatry 1998; 65: 5736.

11. Braekken SK, Russell D, Brucher R, Abdelnoor M, Svennevig JL. Cerebral microembolic signals during cardiopulmonary bypass surgery. Frequency, time of occurrence, and association with patient and surgical characteristics. Stroke 1997; 28: 1988-92.

12. Rodriguez RA, Belway D. Comparison of two different extracorporeal circuits on cerebral embolization during cardiopulmonary bypass in children. Perfusion 2006; 21: 247-53. 